\title{
The problem of forecasting emergency situations of technical objects
}

\author{
Ekaterina Sokolova, ${ }^{1, *}$ and Andrey Sokolov ${ }^{2}$ \\ ${ }^{1}$ Federal State Autonomous Institution «Military Innovation Technopolis «ERA» Anapa, prospect \\ Pionersky 28, Russia \\ ${ }^{2}$ North Caucasian Institute of Mining and Metallurgy (State Technological University), 362021, \\ North Ossetia-Alania Vladikavkaz, street Nikolaeva, 44, Russia
}

\begin{abstract}
The problem of edge state forecasting has a wide field of application. To begin with, it arises when one develops a technical diagnostics system as the problem of forecasting emergency situations of technical objects. When the ecological system is concerned it appears as the problem of forecasting unfavorable development of the ecological situation. In case of investment analysis it evolves as the problem of forecasting the risks of no profit. In medical diagnostic automated systems it is the forecasting disease progression and transition.
\end{abstract}

\section{Introduction}

In general, the problem of forecasting edge state emerges in the situation of developing systems of automatic control of any complex objects and is used to identify critical situations that result from inadequate dynamics of the object under test. The aim of optimal solution of the edge state forecasting problem is to enhance complex systems' reliability.[1]

There are several main phases in the process of solving the problem of edge states forecasting.

The first phase solves the problem of identifying the set of prognostics with low level of redundancy and high information content. On the prognostic procedures development stage mathematic simulation of edge state risk assessment is carried out to define edge overrunning probability.

While solving the problem of the edge state forecasting an important role belongs to the full-scale experiment phase. Herein, as a rule its cost and duration become the main components of the general costs and time necessary to create a complex system. At this point there is still a task of developing exact and approximate algorithms of solving multiobject problem of full-scale experiment hardware and software reliability enhancement as well as a task of virtual backup element modeling.[2]

To solve the task at hand we have carried out mathematical method analysis of modeling digital methods of extremum seeking in the complex system edge state forecasting optimization. The process of edge state forecasting system development is presented as a stage sequence in Fig. 1.

\footnotetext{
*Corresponding author: katya_sea@mail.ru
} 


\section{Materials and Methods}

We have provided mathematical simulation task overview for each of these phases and listed the main mathematical simulation and edge state forecasting computer optimization method technical problems.[3]

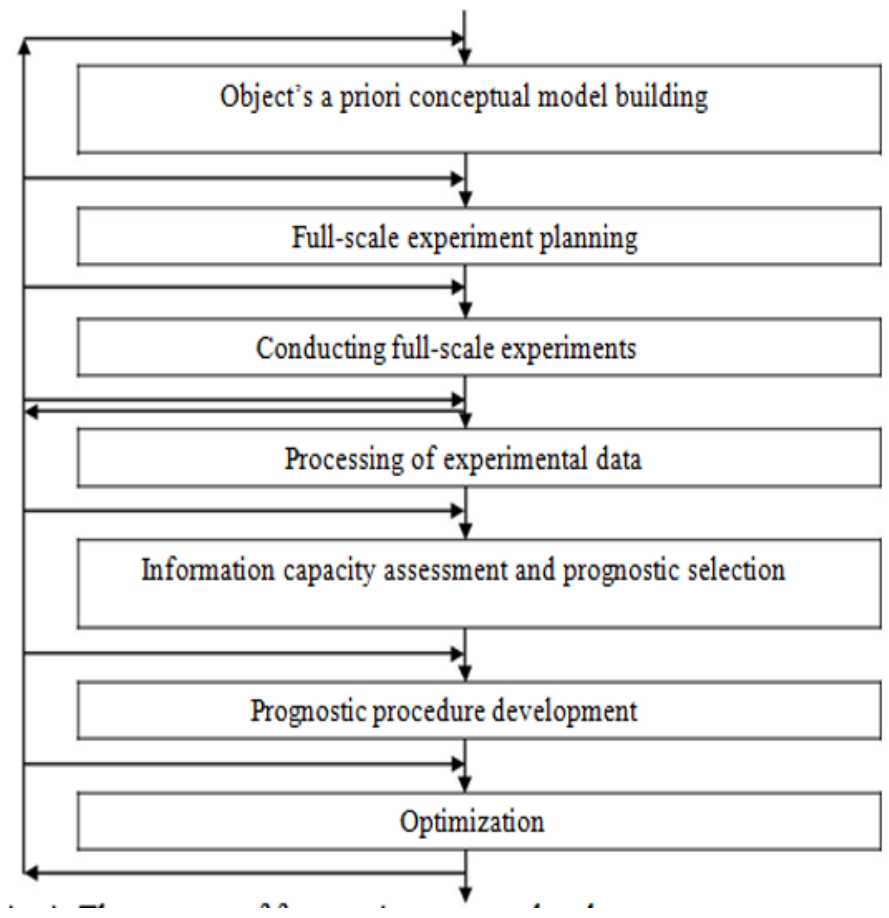

Fig. 1. The process of forecasting system development.

It is shown that he following tasks in the edge state forecasting system development need to be optimized: informative prognostics development and selection, object's edge state risk forecast, edge state forecasting system reliability enhancement at the full-scale experiment phase, planning the object's initial condition recovery upon full-scale experiment completion.

We have outlined the complexity and cohesion of these modelling and digital optimization tasks in the process of forecasting and uniting solution methods into dual computing environment based on using optimization and neural network modelling. The dual approach presupposes that the synergetic combination of neural network and optimization models will let to enhance prognostic algorithms' computing capability[4].The integration of optimization and neural network methods enables the usage of individual characteristics of each method to solve specific task components[5]. As a result the forecast task can be examined from different perspectives, which will give more accurate and unbiased result. The proposed dual computing environment structure is shown in Fig. 2.

Then we carried out mathematical simulation of assessment of the edge state risks, connected with the possibility of overrunning these edges. We have studied the complex system whose controlled variable within the set time should reach the target value; at this the rate of change of the given parameter should stay within certain tolerable limits. 


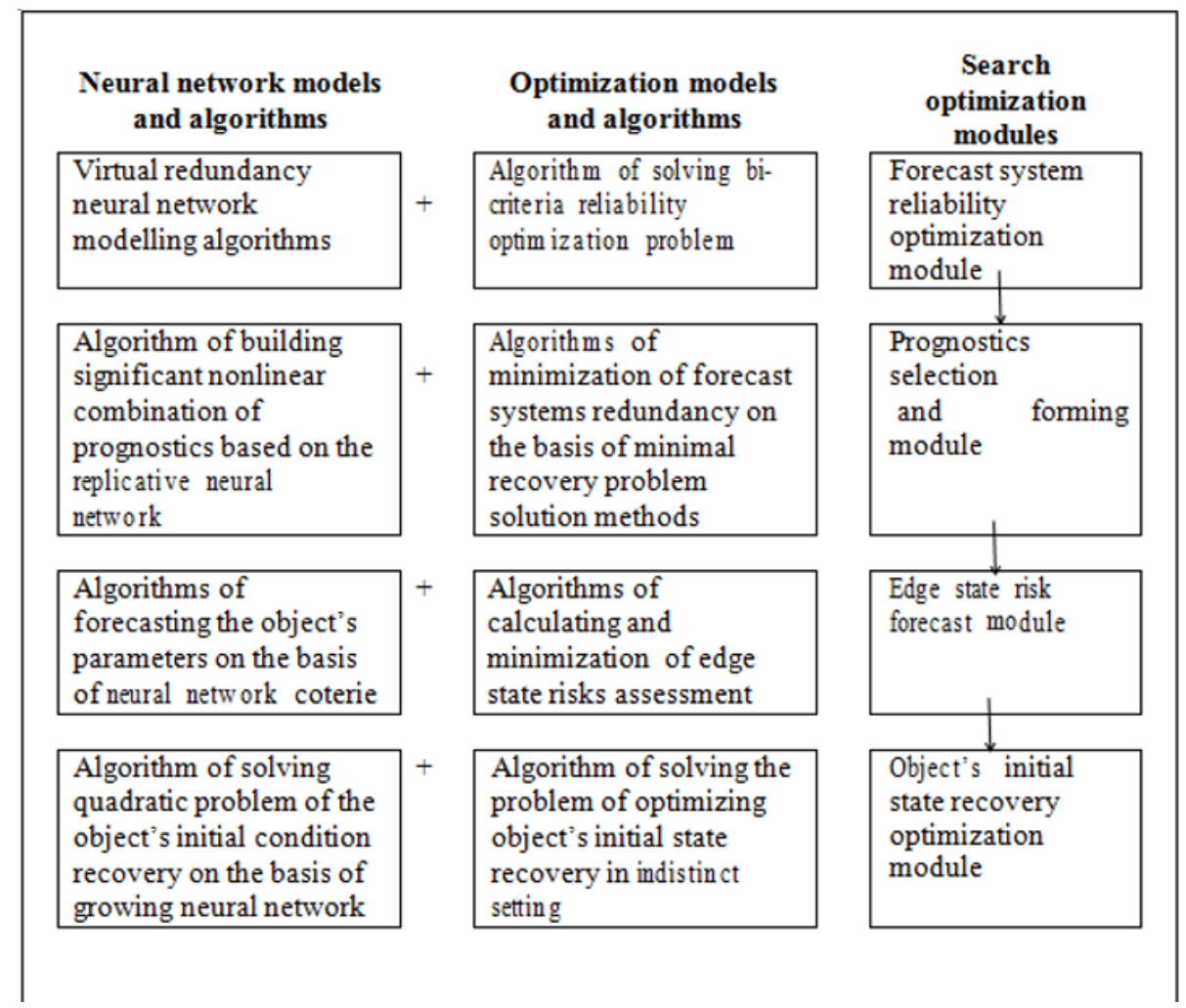

Fig. 2. Dual computing environment structure.

In this context, the risk is defined as the probability of not-reaching the stated objective due to overrunning the tolerable edges. The risk is seen as a variable representing a functional relation to the instantaneuos value of the measured quantity. The risk increases as the system parameters approach the tolerable edges, and after overrunning those edges the system becomes uncontrolled.

The strategy of building and traversing the decision tree

As the simulation experiment has shown, the most significant enumeration reduction is provided by examining coordinates in the order of decreasing the value of the relation $\mathrm{s} j / \mathrm{p}$ $\mathrm{j}$. Preliminary element arrangement in the order of $\mathrm{s} \mathrm{j} / \mathrm{p} \mathrm{j}$ coefficient decreasing and the consequent decision tree bifurcation according to the acquired succession is an effective way to increase the solution search rate for the edge state forecast system reliability enhancement by means of the proposed branch and bounds method. 


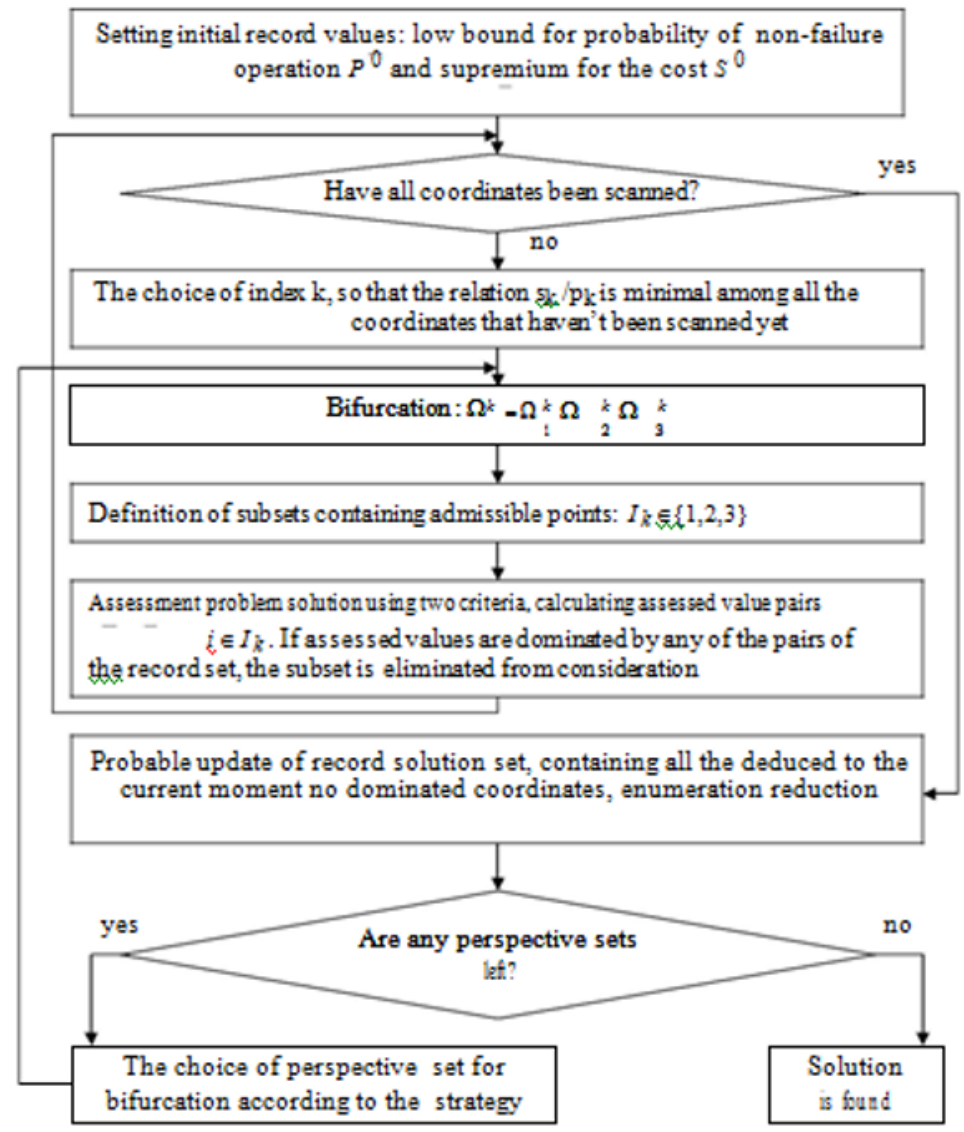

Fig. 3. Flow-chart of the branch and bounds method.

\section{Results}

As can be seen from the above, we propose the branch and bounds method, whose distinctive feature is the search of complete collection of Pareto optimal solution of a bicriteria problem.

The basic expected result - working out of technologies for the assessment and the forecast of emergency technological situations development, dynamics and scale of their development on the basis of joint use of methods of the technical control system enhancement and mathematical modeling.

\section{Discussion}

By means of the multi-criteria optimization model of the algorithm that has been developed for it we have solved the problem of the technical control system reliability enhancement. Among the 46 Pareto optimal solutions that have been found by the branch and bounds method we have chosen the result that meets the technical specifications requirements of reliability index 0.99 during 10000 hours (one-component system had reliability index of 0.9). One of the main criteria of the compromise decision choice was the fact that technical implementation of such reserved structure does not involve any problem. 


\section{References}

1. Zhi-liang Zhu, Wei Zhang, Kwok-wo Wong and Hai Yu Inf. Sci. 6 1171-86 (2011)

2. Wei Zhang, Kwok-wo Wong, Hai Yu and Zhi-liang Zhu Commun. Nonlinear Sci. Numer. Simul. 3 584-600 (2013)

3. Avdeyev B Masyutkin E Golikov S Sokolov S and V. Gavrilov 2017 IEEE Conference of Russian Young Researchers in Electrical and Electronic Engineering (EIConRus) 1225-1228 (2017)

4. Avdeyev B MATEC Web Conf. International Conference on Modern Trends in Manufacturing Technologies and Equipment (ICMTMTE 2017) 129 06012(2017)

5. $\quad$ Xingyuan Wang, Lin Teng and Xue Qin 2012 Signal Process 4 1101-08 (2012)

6. $\quad$ Yicong Zhou, Weijia Cao, and Philip Chen Signal Process 8 197-207 (2014)

7. Murillo-Escobar M A, Cruz-Hernández C, Abundiz-Pérez F López-Gutiérrez R M and Acosta Del Campo O R Signal Process 109 119-131 (2015)

8. Sokolova E.A MATEC Web of Conferences 1295 (2017)

9. Sokolova E.A. Dzhioev G.A IOP Conference Series: aterials Science and Engineering 177 5(2017)

10. Yingqian Zhang and Xingyuan Wang Inf. Sci. 20 329-351 (2014)

11. Wei Zhang, Kwok-wo Wong, Hai Yu and Zhi-liang Zhu Commun. Nonlinear Sci. Numer. Simul. 8 2066-80 (2013) 